\title{
Multichannel semiconductor-based electrodes for in vivo electrochemical and electrophysiological studies in rat CNS
}

\author{
Craig G. van Horne ${ }^{1}$, Spencer Bement ${ }^{3}$, Barry J. Hoffer ${ }^{1,2}$ and Greg A. Gerhardt ${ }^{1,2}$ \\ Departments of 'Pharmacology and 'Psychiatry, University of Colorado Health Sciences Center, Denver, CO 80262 (U.S.A.) and ${ }^{3}$ Department of \\ Electrical Engineering and Computer Science, University of Michigan, Ann Arbor, MI 48109 (U.S.A.)
}

(Received 23 April 1990; Revised version received 23 August 1990; Accepted 24 August 1990)

Key words: In vivo electrochemistry; Multichannel microelectrode; Electrophysiology; Nafion; Monoamine; Cerebellum

\begin{abstract}
Five-channel silicon-based microprobes were sputter-coated with carbon, coated with Nafion, and used for both in vivo electrochemical and singleunit electrophysiological recordings. High-speed electrochemical studies were performed in vitro and in vivo, which demonstrated that these multisite probes were capable of monitoring the evoked overflow of monoamines in selected brain regions of the rat. In addition, action potentials from Purkinje cells in the rat cerebellum, identified electrophysiologically, were recorded from different sites on the same probe. Spontaneous firing rates could be monitored for up to 2 hours in order to investigate the effects of systemic administration of phencyclidine. These results provide preliminary evidence that solid-state multi-site probes can be utilized for both in vivo electrochemical and electrophysiological studies in the rat brain.
\end{abstract}

Neural network theory [1] has provided a conceptual framework for mechanisms of learning [11], memory [10], sensory discrimination $[4,6]$, and other 'higher neural' activities. Artificial neural network models [3, 10] have been related to components of natural neuronal circuits. Verification of the applicability of these models to neural function, however, demands simultaneous recordings from ensembles of central neurons during behavioral activities, together with measurements of presynaptic dynamics.

A number of approaches to simultaneous recordings of central units have been attempted, including the implantation of large diameter $(62-125 \mu \mathrm{m})$ single wires [19], bundles of smaller diameter $(25 \mu \mathrm{m})$ microwires [12], and semiconductor microprobes $[5,13,18]$. The latter types of electrodes have several theoretical advantages for simultaneous electrophysiological recording of multiple units, especially if it is desired to combine this recording with high-speed in vivo electrochemical measurements of transmitter overflow during the same realtime epoch [9]. These advantages would include precise spatially resolved multiple recording sites, reproducible electrophysiological and electrochemical recording surfaces, and increased mechanical strength. In this study

Correspondence: G.A. Gerhardt, Dept. of Psychiatry, Box C268, University of Colorado, Health Sciences Center, 4200 East Ninth Ave., Denver, CO 80262, U.S.A. we had two objectives. First, we sought to characterize the ability of the multi-channel semiconductor recording electrodes to measure monoamines both in vitro and in vivo using high-speed electrochemical recording techniques. Secondly, we wished to test whether the probes could be used for electrophysiological recordings and to compare the recorded signals with those obtained with glass microelectrodes.

The multichannel ( 5 recording sites per sensor) semiconductor-based probes were fabricated by the Center for Integrated Sensors and Circuits, at the University of Michigan, Ann Arbor [2]. The probes were fabricated using the high-yield approaches developed by Najafi et al. [15]. Fig. 1A shows the basic design characteristics of the probes. The 5 individual recording sites were sputtercoated with $500 \mathrm{~nm}$ of carbon. The electrodes were then wire-bonded to a circuit board carrier for the acute recordings in anesthetized animals (see below) and were dip-coated with Nafion [7-9] to convert them into voltammetric recording sensors. Four different designs were employed for the present investigations, which varied in recording site area. The electrode recording sites on the silicon substrate were each positioned $200 \mu \mathrm{m}$ from the center of the adjacent site in series, and the 5-channel electrodes spanned a recording area of $1 \mathrm{~mm}$ in all 4 electrode designs. The total surface areas of each type multichannel probe were $1000(n=2), 2000(n=2), 4000$ $(n=4)$, and $8000 \mu \mathrm{m}^{2}(n=5)$ per recording site. All 4 of the probe designs were used for high-speed electrochemi- 
A.

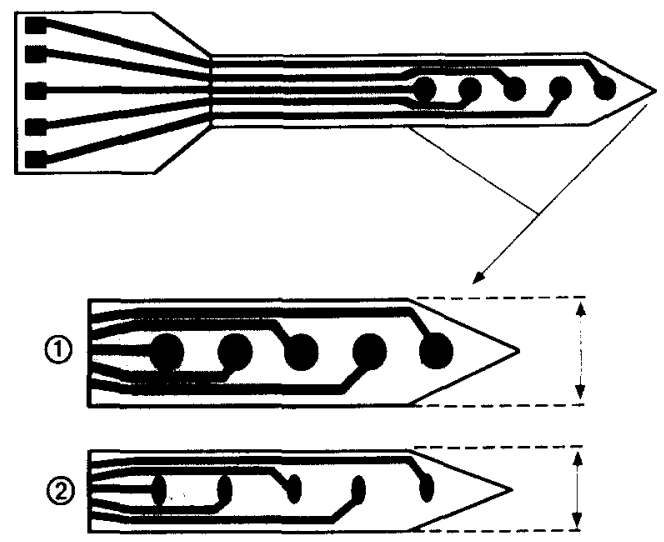

B.

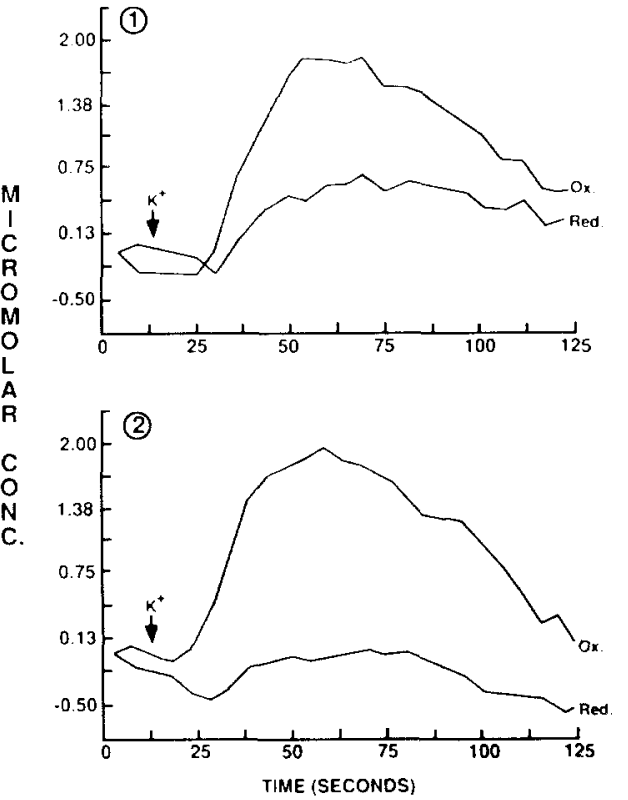

Fig. 1. A: illustration of the various types of multichannel ( 5 site) recording electrodes used in this study. Type 1 electrodes had shank widths of 200 or $250 \mu \mathrm{m}$ with recording sites of 4000 and $8000 \mu \mathrm{m}^{2}$, while type 2 probes had shank widths of 150 and $180 \mu \mathrm{m}$ with recording sites of 1000 and 2000 , respectively. B: high-speed in vivo electrochemical recordings of potassium-evoked overflow of monoamines in the medial prefrontal cortex of the rat. The ejections of potassium are indicated by the arrows.

cal investigations while only the 1000,2000 , and 4000 $\mu \mathrm{m}^{2}$ surface area electrodes were employed for both electrochemical and electrophysiological investigations (see below).

Male Sprague-Dawley Rats (250-300 g) were anesthetized with urethane $(1.25 \mathrm{~g} / \mathrm{kg})$, intubated, and placed in a stereotaxic frame for both in vivo electrochemical and electrophysiological studies. Electrochemical recordings were performed in the rat striatum and medial prefrontal cortex as determined by stereotaxic electrode placement [17]. All in vivo electrochemical measurements were performed using a high-speed chronoamperometric recording system (IVEC-5; Medical Systems Corp.). The multichannel probes were characterized for sensitivity to dopamine, norepinephrine and serotonin in vitro, and their selectivities to these compounds versus ascorbic acid were characterized. The sensitivities and linear recording characteristics of all recording electrodes were determined by measuring responses to stock solutions of $\mathrm{DA}$; releases were expressed quantitatively in terms of the DA calibration curves. Square-wave pulses of 0.0 to $+0.55 \mathrm{~V}$, with respect to $\mathrm{ag} / \mathrm{AgCl}$ reference electrode, were applied to the working electrode for $0.20-1.0 \mathrm{~s}$. The resulting oxidation and reduction currents were digitally integrated during the final $60-80 \%$ of the recording period; one digital count recorded was approximately equal to $1.7 \times 10^{-13} \mathrm{C}$. Releases of monoamines were elicited by local application of $\mathrm{K}^{+}(120 \mathrm{mM} \mathrm{KCl}$ and $2.5 \mathrm{mM}$ $\mathrm{CaCl}_{2} ; 100-250 \mathrm{nl}$ ejected) from micropipettes having outer tip diameters of 5-10 $\mu \mathrm{m}$ [8]. These were attached to the electrochemical recording electrodes using wax with a tip separation of $275-350 \mu \mathrm{m}$. In addition, extracellular single-unit recordings of cerebellar Purkinje neurons in the anesthetized rat were obtained with the multisite Nafion-coated probes and with conventional singlebarrel glass micropipettes as previously described [14, 16].

The recording sites of all 4 probe designs, following dip-coating with Nafion, responded linearly to increasing concentrations of dopamine; the linear regression correlation coefficients of the calibration curves were greater than 0.997 ( $n=8$ probes). In addition, some probes were also tested for their sensitivity to norepinephrine and serotonin, and were seen to respond linearly to these compounds as well. In addition, good selectivities for the detection of dopamine versus ascorbic acid (AA) were achieved. Typical selectivities for DA versus AA ranged from 100 to $700: 1$ and averaged $261 \pm 106$ (S.E.M., $n=5$ ) for the $8000 \mu \mathrm{m}^{2}$ area probes. However, the charging currents of these probes were often higher which resulted in the necessity to use lower gain settings on the current to voltage amplifiers. As compared to carbon epoxy or carbon fiber electrodes we have previously studied [7-9], the signal-to-noise characteristics were also lower. For example, our graphiteepoxy electrodes, which have a surface area close to 8000 $\mu \mathrm{m}^{2}$ (geometric area based on a disk surface $100 \mu \mathrm{m}$ in diameter), record signals of $5385 \pm 259$ (S.E.M., $n=22$ ) digital counts per micromolar change in DA (one digital account is approximately equal to $1.7 \times 10^{-13} \mathrm{C}$ ). This is 

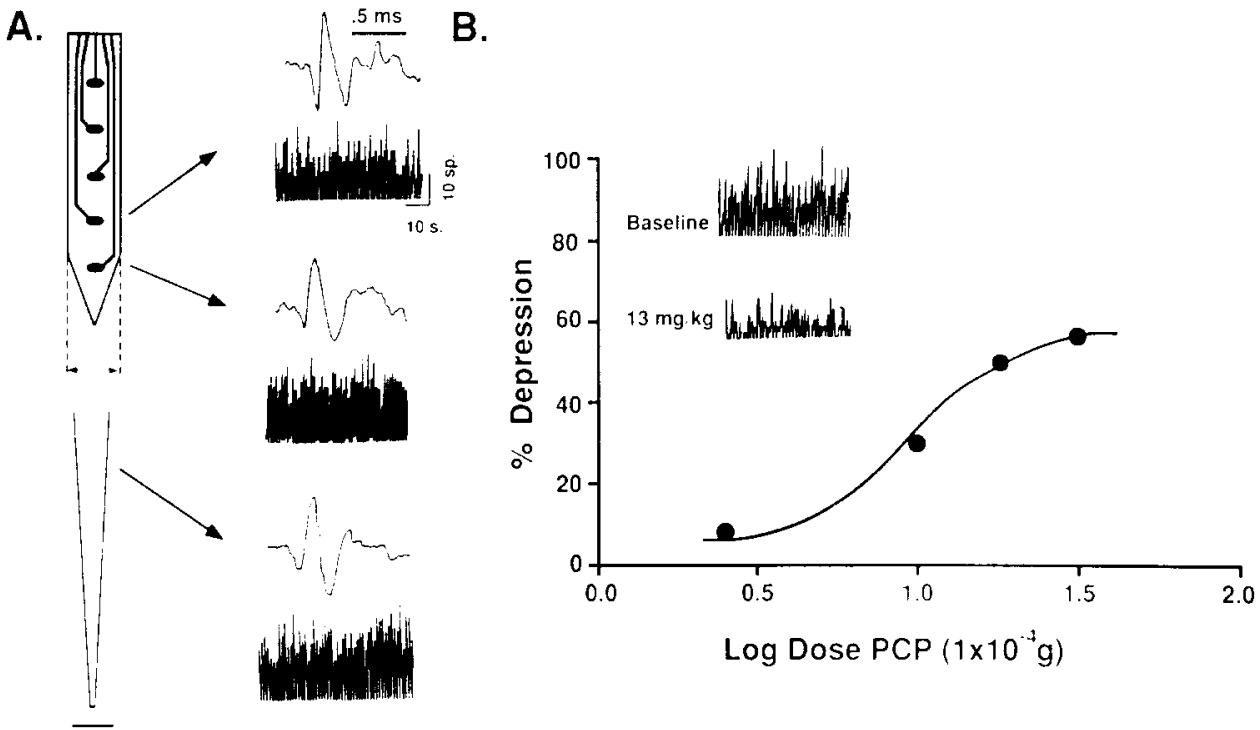

Fig. 2. A: electrophysiological recordings with a multichannel semiconductor electrode $\left(2000 \mu \mathrm{m}^{2}\right.$ surface area per site; width of electrode shank was $180 \mu \mathrm{m})$ and a conventional glass micropipette $(\mathrm{bar}=10 \mu \mathrm{m})$. Two different cerebellar Purkinje neurons obtained from the lower two sites were recorded from the probe. B: demonstration of the ability of a multichannel electrode ( $4000 \mu \mathrm{m}^{2}$ recording area per site) to record the sustained activity of a cerebellar Purkinje neuron and cumulative dose-response curves from the systemic administration of PCP. The two insets show portions of the actual ratemeter tracings before and $5 \mathrm{~min}$ after the systemic administration of $13 \mathrm{mg} / \mathrm{kg} \mathrm{PCP}$.

in contrast to the $821 \pm 80(n=5)$ counts per micromolar change in DA seen for the $8000 \mu \mathrm{m}^{2}$ recording sites of the semiconductor probes. In addition, the reduction current responses recorded by these probes were consistently lower than the signals recorded using carbonbased recording electrodes [9]. Typical reduction/oxidation current responses for DA using the $8000 \mu \mathrm{m}^{2}$ area probes averaged $0.31 \pm 0.04$ (S.E.M., $n=5$ ) at a $5 \mathrm{~Hz}$ recording rate, as compared to $0.58 \pm 0.02 \quad(n=22)$ recorded with standard carbon epoxy electrodes [9].

Individual electrochemical recordings, using multichannel (8000 or $4000 \mu \mathrm{m}^{2}$ recording sites) semiconductor-based probes, of monoamine overflow produced by local application of potassium were recorded in striatum and the medial prefrontal cortex of 2 anesthetized rats (Fig. 1B). The 'Ox' curves (upper traces) represent the high-speed oxidation current recordings while the 'Red' tracings (lower traces) are the reduction current responses. Signal 1 was recorded $1.0 \mathrm{~mm}$ from cortical surface while signal 2 was recorded at a depth of $2.0 \mathrm{~mm}$. In one animal, the signals recorded from the prefrontal cortex averaged $2.2 \pm 0.2 \mu \mathrm{M}(n=4)$ in amplitude; values were expressed in DA equivalents using calibration factors determined for each probe in vitro. These signals and oxidation/reduction ratios are quite similar to those recorded in this same region with conventional graphite epoxy electrodes [9].

Nafion-coated probes were also used for electrophysiological recording before or after the electrochemical studies ( $n=4$ animals). Insertion of the probe into the cerebellum far anterior or posterior in the folium, where
Purkinje cells are aligned vertically, often yielded recordings from such neurons at two or more sites on the same probe (Fig. 2A); sites were selected using an electrode switching circuit connected to a single-channel recording amplifier. Action potentials from different neurons at two recording sites were seen simultaneously on a number of occasions ( 2 animals). Purkinje cells were identified by their firing rates (range $=6-30 \mathrm{~Hz}, n=12$ cells) in conjunction with their characteristic firing patterns composed of both simple and complex spikes [14]. The firing rates and waveform shapes recorded from the multichannel probes were similar to those recorded by a conventional glass micropipette in the same folium (Fig. 2A).

The ability of the probe to record sustained electrophysiological activity, and its alteration by pharmacological manipulation of presynaptic input, was investigated in 2 animals and such data are shown in Fig. 2B. In this experiment, activity from a cerebellar Purkinje neuron was continuously recorded for over two hours. During this time, phencyclidine (PCP) was administered parenterally in increasing doses, which caused dose-dependent decreases in the basal Purkinje cell firing rate; individual action potentials were periodically viewed to ensure that the effects of PCP were not due to local anesthetic effects or movement of the recording electrodes. In the cerebellum, PCP acts as an indirect noradrenergic agonist, probably through inhibition of the uptake carrier for norepinephrine [14].

This study provides the first documentation that Nafion-coated semiconductor-based multichannel 
recording electrodes can be used to quantitatively measure monoamines both in vitro and in vivo using highspeed chronoamperometric recording techniques, and that the same multichannel probes can be used for single-unit electrophysiological recordings. Moreover, electrophysiology experiments that require long recording times, such as those analyzing drug dose-effect relationships, can be investigated with these probes.

Dip-coating of the multichannel probes with Nafion was seen to increase the selectivity of the recording sites for cationic neurotransmitters versus anionic interferents such as ascorbic acid. The selectivities of the individual probes for DA were similar to those seen using Nafioncoated graphite epoxy capillary or carbon fiber electrodes [7-9]. In addition, the Nafion coating did not appear to negatively influence the multichannel recording probes for single-unit electrophysiological measurements, as the recording characteristics of these sensors were seen to rival those seen with more standard glass micropipette electrodes. Interestingly, preliminary attempts to use the multichannel electrodes without the Nafion coating were relatively unsuccessful. Single Purkinje cells were not easily identified, and the signal-tonoise properties of the electrodes appeared to be significantly compromised. Thus, the Nafion coating may have a positive effect on the electrophysiological recording properties of the multichannel probes. In addition, the potential utilization of Nafion coatings to improve the electrophysiological recording properties of other metal recording electrodes should be investigated.

There are several additional technological advances that must be made before such probes will be truly useful to characterize the emergent properties of neural ensembles in behaving animals. First, the recording surfaces need to be improved for combined electrochemical and electrophysiological recordings. The type and thickness of carbon layer on the surface must be optimized, as well as the procedures for coating the probes with Nafion. Secondly, microdrives and wire-bonding techniques must be developed to allow implantation of such probes into awake behaving animals. Third, computer interfacing and hardware must be fabricated to allow simultaneous acquisition and storage of multiple single-unit electrophysiological and multichannel electrochemical signals. The cost of such hardware must be within the reach of individual laboratories. Finally, analytical schemes must be formulated and translated into realtime software, in order to quantitatively analyze such large volumes of data and to test hypotheses of neural network function. These complexities notwithstanding, the data in this paper suggest that semiconductor microprobes may play increasingly important roles as realtime biological sensors to analyze simultaneous pre- synaptic neurotransmitter dynamics and activity of multiple neurons.

Supported by USPHS Grants AG-06434, AG-04418, AG-00441 and NS-09199, N01-NS-42364 and the NIMH.

1 Anderson, J.A. and Rosenfeld, E., Neurocomputing: Foundations of Research, MIT Press, Cambridge, MA, 1988.

2 BeMent, S.L., Wise, K.D., Anderson, D.J., Najafi, K. and Drake, K.L., Solid-state electrodes for multichannel multiplexed intracortical neuronal recording, IEEE Trans. Biomed. Eng., 2 (1986) 230 241

3 Buhman, J. and Shulten, K., Associative recognition and storage in a model network of physiological neurons, Biol. Cybern., 56 (1986) 313-327.

4 Carpenter, G.A., Neural network models for pattern recognition and associative memory, Neural Networks, 2 (1989) 243-257.

5 Eichenbaum, H. and Kuperstein, M., Extracellular neural recording with multichannel microelectrodes, J. Electrophysiol. Tech., 13 (1986) 189-209.

6 Fukishima, K., Neocognitron: a hierarchical neural network capable of visual pattern recognition, Neural Networks, 1 (1988) 119 130.

7 Gerhardt, G.A., Oke, A.F., Nagy, G., Moghaddam, B. and Adams, R.N., Nafion-coated electrodes with high selectivity for CNS electrochemistry, Brain Res., 290 (1984) 390-395.

8 Gerhardt, G.A., Rose, G.M. and Hoffer, B.J., Release of monoamines from striatum of rat and mouse evoked by local application of potassium: Evaluation of a new in vivo electrochemical technique, J. Neurochem., 46 (1986) 842-850.

9 Gratton. A., Hoffer, B.J. and Gerhardt, G.A., In vivo electrochemical determination of monoamine release in the medial prefrontal cortex of the rat, Neuroscience, 29 (1989) 57-64

10 Hopfield, J.J., Neural networks and physical systems with emergent collective computational abilities, Proc. Natl. Acad. Sci. U.S.A., 79 (1982) 2554-2558.

11 Kohonen, T., Adaptive, associative and self-organizing functions in neural computing, Applied Optics, 26 (1987) 4910-4918.

12 Kubie, J.L., A driveable bundle of microwires for collecting singleunit data from freely-moving rats, Physiol. Behav., 32 (1984) 115 118.

13 Kuperstein, M. and Whittington, D.A., A practical 24 channel microelectrode array for neural recording in vivo, IEEE Trans. Biomed. Eng., 28 (1981) 288-293.

14 Marwaha, J., Palmer, M., Hoffer, B., Freedman, B., Rice, K.C., Paul, S. and Skolnick, P., Differential electrophysiological and behavioral responses to optically active derivatives of phencyclidine, Naunyn-Schmiedeberg's Arch. Pharmacol., 315 (1981) 203-209.

15 Najafi, N. and Wise, K.D., A high-yield IC-compatible multichannel recording array, IEEE Trans. Electron Devices, 32 (1985) 1206 1211.

16 Palmer, M.R., van Horne, C.G., Harlan, T.J. and Moore, E.A., Antagonism of ethanol effects on cerebellar Purkinje neurons by the benzodiazepine inverse agonist Ro 15-4513 and FG 7142: electrophysiological studies, J. Pharmacol. Exp. Ther., 247 (1988) 1018-1024

17 Paxinos, G. and Watson, C., The Rat Brain in Stereotaxic Coordinates, Academic Press, New York, 1982.

18 Prohaska, O., Pacha, F., Pfundner, P. and Petsche, H., A 16-fold semi-microelectrode for intracortical recording of field potentials, Electroenceph. Clin. Neurophysiol., 47 (1979) 629-631.

19 Strumwasser, F., Long-term recording from single neurons in brain of unrestrained mammals, Science, 127 (1958) 469-470. 